\title{
ON THE SIGNIFICANCE OF NORMAL STRESS EFFECTS IN THE FLOW OF GLACIERS
}

\author{
By ChI-SING MaN \\ (Department of Mathematics, University of Kentucky, Lexington, \\ Kentucky 40506-0027, U.S.A.) \\ and QUAN-XIN SUN \\ (Department of Civil Engineering, University of Manitoba, Winnipeg, \\ Manitoba R3T 2N2, Canada)
}

ABSTRACT. McTigue and others (1985) identified a possible problem in the type of constitutive equation usually used for modeling the creep behaviour of polycrystalline ice. They pointed out that Glen's flow law necessarily excludes the consideration of normal stress effects, which are of great significance in other disciplines that consider nonNewtonian fluids. Using the second-order fluid (with material parameters evaluated from laboratory data) as a tentative model for ice, they reached the conclusion that normal stress effects may be discernible in natural glacier flow. But, as noted by McTigue and others, the secondorder fluid "fails to represent the non-linear rate dependence of ice in shear"; therefore it is in fact not a suitable constitutive model for glacier ice in shearing flow. In this note, parallel to what McTigue and others did for the second-order fluid, we present a similar analysis for (I) the modified second-order fluid and (II) the power-law fluid of grade 2, both of which are constitutive models recently proposed by Man as a tentative generalization of Glen's flow law. Both models (I) and (II) can represent normal stress effects, and both agree with Glen's flow law in the prediction of the depth profile of velocity in the steady laminar flow of glaciers. For ease of comparison, the same creep data of McTigue and others are used in quantifying the material parameters in these two models. Both models (I) and (II) predict far less pronounced normal stress effects in glaciers than those estimated by McTigue and others (whose data analysis in fact suffered from inconsistencies and over-parameterization).

\section{INTRODUCTION}

McTigue and others (1985) studied the possibility of normal stress effects in the creep of polycrystalline ice. Normal stress effects are phenomena that follow from nonzero and unequal normal stress differences in shearing flow; they are among the first manifestations of non-linearity in many fluids. McTigue and others criticized Glen's flow law, because it necessarily excludes consideration of normal stress effects. They adopted as a tentative model for ice the second-order fluid (i.e. one of the simplest models that can represent normal stress effects) and estimated the three material parameters in the model from data of four triaxial creep tests. (Their creep tests were performed at the same temperature and the same deviatoric stress, but at different confining pressures. Here we follow common usage and call those creep tests "triaxial". McTigue and others used the adjective "biaxial".) McTigue and others reported that the resulting model gave an excellent representation of their data for both primary and secondary creep. Using the same model, they reached the conclusion that normal stress effects could influence the development of crevasses and could lead to measurable depression of the surface of glaciers on sufficiently steep slopes.
As noted by McTigue and others, the second-order fluid is in no way a replacement for, nor a generalization of, Glen's $(1952,1955)$ flow law. Indeed, the second-order fluid "fails to represent the non-linear rate dependence of ice in shear"; as such, it is in fact not a suitable constitutive model for glacier ice in shearing flow. Take, for instance, Nye's first model of a glacier as an infinite slab of ice of uniform thickness flowing down a rough inclined plane (Nye, 1952, 1957). Consider steady laminar flow. For a slab of second-order fluid, the velocity field will be identical to that of an incompressible linearly viscous fluid. Such an implication is certainly undesirable. Since McTigue and others used the second-order fluid to model ice when they discussed the possible significance of normal stress effects in the flow of glaciers, the conclusion they reached, namely that normal stress effects may be discernible in natural glacier flow, is questionable.

Glen's flow law, as extended to cover multi-axial states of stress by Nye $(1953,1957)$, in effect models polycrystalline ice as an incompressible power-law fluid (cf. Bird and others, 1977, p. 208). It is the most widely used empirical flow law for polycrystalline ice under stress. While its successes are well documented (Hooke, 1981), it has its shortcomings, namely: it can only describe secondary creep, and it does not predict any normal stress effect in shearing flow of ice (cf. McTigue and others (1985) for a more detailed criticism).

Motivated by a preliminary version of the paper of McTigue and others, Man and others (1985) initiated a research program in 1984, the purpose of which has been to seek a simple modification of Glen's flow law that obviates the aforementioned shortcomings. Based on results of pressuremeter creep tests performed by $\mathrm{K}$ jartanson and Shields at the University of Manitoba (K jartanson, unpublished) and with the help of Sun in numerical work, Man has proposed two tentative modifications of Glen's flow law. The purpose of the present note is to re-examine the issues discussed by McTigue and others (1985) when the secondorder fluid in their paper is replaced by the two new tentative models (I) and (II). For ease of comparison, material parameters in models (I) and (II) will be estimated by fitting data of the four triaxial creep tests of McTigue and others.

The specific choice of tentative models (I) and (II) was mainly guided by empiricism and the requirement of simplicity. We want to seek a simple model which will combine the merits of the partially successful power-law and second-order fluid models. Compared with the secondorder fluid, which follows from the rational approximation scheme of Coleman and Noll (1960), some readers will find models (I) and (II) much less appealing to their intellect. All mathematical theorems, however, are logical deductions from specific assumptions. The assumptions upon which the approximation scheme of Coleman and Noll is based need not apply to the creep of polycrystalline ice. And 
apparently they do not, as we can gather from what is known about the creep behavior of ice. In the history of physics, it is more common than otherwise that empirical laws were discovered long before any rationale could be found to justify their existence.

In this note, by Glen's flow law we shall mean either the formulation in terms of "octahedral strain-rate" and octahedral shear stress (see Equation (5) below) or the generalization due to Nye $(1953,1957)$, i.e. the power-law fluid model, which is sometimes known as "the generalized flow law" in the literature (e.g. Paterson, 1981, p. 30). It should be clear from the context which of the two versions we really mean when we refer to Glen's flow law below.

\section{TWO MODIFICATIONS OF NYE'S GENERALIZATION OF} GLEN'S FLOW LAW

In what follows we shall, with few exceptions, adopt the notation of McTigue and others (1985). Tensors will be denoted by Cartesian indicial notation. To conserve space, we shall not reproduce definitions of terms such as normal stress differences and Rivlin-Ericksen tensors, which are given in the paper of McTigue and others and in texts of non-Newtonian fluid mechanics (e.g. Schowalter, 1978).

Man has proposed the following two constitutive equations as tentative modifications of "the generalized flow law":

(I) $T_{i j}+p \delta_{i j}=\mu \Pi^{m / 2} A_{i j}^{(1)}+\alpha_{1} A_{i j}^{(2)}+\alpha_{2} A_{i k}^{(1)} A_{k j}^{(1)}$,

(II) $T_{i j}+p \delta_{i j}=\Pi^{m / 2}\left(\mu A_{i j}^{(1)}+\alpha_{1} A_{i j}^{(2)}+\alpha_{2} A_{i k}^{(1)} A_{k j}^{(1)}\right) ;(2)$

here $T_{i j}$ is the Cauchy stress tensor; $p$ is the indeterminate pressure due to incompressibility; $A_{i j}^{(1)}$ and $A_{i j}^{(2)}$ are the first and the second Rivlin-Ericksen tensor, respectively; $A_{11}^{(1)}+A_{22}^{(1)}+A_{33}^{(1)} \equiv 0$ because of incompressibility; $\Pi \equiv \frac{1}{2} A_{j k}^{(1)} A_{k j}^{(1)} ; \quad \mu, \quad \alpha_{1}, \quad \alpha_{2}$, and $m$ are material parameters, which are constants for polycrystalline ice of given temperature and given structural features such as texture and fabric; the parameter $m$ is related to the exponent $n$ in Glen's flow law by $n=1 /(m+1)$ (thence $n=3$ corresponds to $m=-2 / 3$ ). When $\alpha_{1}=\alpha_{2}=0$, both models (I) and (II) reduce to the power-law fluid, i.e. Glen's flow law. When $m=0$, both models (I) and (II) become the second-order fluid. Both models (I) and (II) belong to a class of fluids introduced by Rivlin and Ericksen (1955); they are special instances of (incompressible) Rivlin-Ericksen fluids of complexity 2 and are formally included in a class of fluids studied by Dunn (1982). They are also special instances of a class of fluids proposed by Morland and Spring (1981) for modeling the deformation of ice. Both models satisfy the principle of material frame-indifference (Truesdell and Noll, 1965, section 19). Both models are meant for the description of primary and secondary creep, but not tertiary creep.

The specific choice of models (I) and (II) is mainly guided by empiricism and the requirement of simplicity. Model (II), however, can be viewed as the second in a hierarchy of more and more complex models, the first or simplest of which is the usual power-law fluid. We shall call it the power-law fluid of grade 2 . In general, by the power-law fluid of grade $k$ we mean the constitutive equation that results when the terms between parentheses in Equation (2) are replaced by terms appropriate to the fluid of grade $k$ in the approximation scheme of Coleman and Noll (1960). The usual power-law fluid is then of grade 1 . We call model (I) the modified second-order fluid.

Both models (I) and (II) gave good fits to data of single-stage and multi-stage pressuremeter creep tests (Man and others, 1985; K jartanson, unpublished), which were performed at $-2{ }^{\circ} \mathrm{C}$ with cavity pressure ranging from 1.0 to $2.5 \mathrm{MPa}$. Between them, model (II) consistently gave better fits. Complete details of fitting have been given by Sun (unpublished).

For steady laminar flow in Nye's $(1952,1957)$ first model of a glacier, both models (I) and (II) deliver a velocity field identical to that which pertains to Glen's flow law. The stress distribution, however, is different for all of the three models.

\section{DETERMINATION OF MATERIAL PARAMETERS}

The material parameters $m, \mu$, and $\alpha_{1}$ in both models (I) and (II) can be determined unambiguously from data of pressuremeter creep tests (Man and others, 1985; K jartanson, unpublished; Sun, unpublished); the parameter $\alpha_{2}$ does not appear in the differential equation that governs pressuremeter creep tests. The purpose of the present note, however, is to re-examine the conclusions of McTigue and others (1985) in the light of models (I) and (II). For comparison purposes, we should determine the material parameters in models (I) and (II) by fitting data of the triaxial creep tests of McTigue and others.

For triaxial creep tests, we shall adopt the same kinematical assumptions as McTigue and others, namely: during the test the cylindrical specimen undergoes a homogeneous, isochoric, uniaxial elongational flow so that its shape always remains cylindrical. We shall neglect body forces and adopt the creeping-flow approximation.

Under the above assumptions it is straightforward to deduce the differential equations that govern triaxial creep tests for models (I) and (II), respectively. Since the derivation is similar to that presented by McTigue and others for the second-order fluid, here we are content to write down the end equations only. For model (I), the differential equation in question is

$$
3 \alpha_{1} \dot{a}+3 \mu\left(3 a^{2}\right)^{m / 2} a+3\left(\alpha_{1}+\alpha_{2}\right) a^{2}-\sigma=0 ;
$$

here a superposed dot denotes differentiation with respect to time; $a=i / l$, where $l(t)$ is the length of the cylindrical specimen at the instant $t ; \sigma$ is the axial stress in excess of the confining pressure. For model (II) or the power-law fluid of grade 2, the creep equation is

$$
3 \alpha_{1} \dot{a}+3 \mu a+3\left(\alpha_{1}+\alpha_{2}\right) a^{2}-\left(3 a^{2}\right)^{-m / 2} \sigma=0 .
$$

When $m=0$, both Equations (3) and (4) reduce to equation (13) of McTigue and others, i.e. the creep equation for the second-order fluid.

Let $\dot{\epsilon} \equiv 3^{-\frac{1}{2}}\left(D_{i j} D_{i j}\right)^{\frac{1}{2}}$ be the "octahedral strain-rate"; here $D_{i j}=\frac{1}{2} A_{l j}^{(1)}$ is the stretching tensor or "strain-rate" tensor. Let $\tau \equiv 3^{-\frac{1}{2}}\left(T_{i j}^{\prime} T_{i j}^{\prime}\right)^{\frac{1}{2}}$ be the octahedral shear stress; here $T_{i j}^{\prime}$ denotes the deviatoric stress tensor. Glen's flow law is often expressed in terms of $\dot{\epsilon}$ and $\tau$ in the form

$$
\dot{\epsilon}=(\tau / B)^{n}
$$

where $B$ and $n$ are material constants (Hooke, 1981). For compression tests, the quantity $a$ in Equations (3) and (4) is related to $\dot{\epsilon}$ by $a=-2^{\frac{1}{2}} \dot{\epsilon}$, whereas $\sigma$ is related to $\tau$ by $\sigma=-\left(3 / 2^{\frac{1}{2}}\right) \tau$. When the absolute value of $a$ is at its minimum $|a|_{\min }, \dot{a}=0$. In order that Equations (3) and (4) should deliver Glen's flow law in secondary creep, the estimates below must be valid for models (I) and (II), respectively:

$$
\begin{aligned}
& \text { (I) }\left|\alpha_{1}+\alpha_{2}\right| \ll 3^{m / 2} \mu|a|_{\min }^{m-1} ; \\
& \text { (II) }\left|\alpha_{1}+\alpha_{2}\right| \ll \mu /|a|_{\min } .
\end{aligned}
$$

Models (I) and (II) are meant to be modifications of Nye's generalization of Glen's flow law. They should be consistent with laboratory data and field measurements which support Glen's flow law. Henceforth we shall assume that estimates (6) and (7) be valid for triaxial creep tests with octahedral shear stresses between 0.1 and $1 \mathrm{MPa}$ (Hooke, 1981). After setting $\dot{a}=0$ and ignoring the term $3\left(\alpha_{1}+\alpha_{2}\right) a^{2}$, both Equations (3) and (4) reduce to the equation

$$
3 \mu\left(3 a^{2}\right)^{m / 2} a=\sigma .
$$

Equation (8) will agree with Equation (5) if and only if

$$
B=2(6)^{m / 2} \mu, \quad n=1 /(m+1) .
$$


All four creep tests of McTigue and others (1985) were done at $\sigma=-0.47 \mathrm{MPa}$ and at essentially the same temperature $\left(-9.5^{\circ} \mathrm{C}\right.$ to $\left.-9.8^{\circ} \mathrm{C}\right)$, but at different confining pressures. Within the framework of either of our models, which assume incompressibility of ice, the four tests would ideally produce the same creep curve should the initial conditions of the tests be identical. With $\sigma$ fixed, tests under different confining pressures will certainly tell us something about the assumption of incompressibility. Besides that, the four tests will have no more information content than repeating one single test four times.

Indeed, we immediately face the problem of overparameterization when we attempt to estimate the material parameters in Equations (3) and (4) by fitting the data of McTigue and others. The parameters are ill-determined because of insufficient data. ${ }^{*}$ Fortunately, we can still proceed after making some reasonable assumptions.

In fitting the data of McTigue and others, our first assumption was that the value of the parameter $m$ was close to $-2 / 3$ (i.e. $n \cong 3$ ). This assumption is in line with Hooke's (1981) conclusion in his review on Glen's flow law.

Only those data (day 1 to day 17) pertaining to primary and secondary creep were used in the fitting. In each test, the initial value of $|a|$ on day 1 was less than $3|a|$ my Equation (8) the value of $|a|_{\min }$ which corresponds to $\sigma=-0.47 \mathrm{MPa}$ will be only about 0.01 times that which corresponds to $\tau=1 \mathrm{MPa}$. Since estimates (6) and (7) should still be valid when $\tau=1 \mathrm{MPa}$, it follows that the term $3\left(\alpha_{1}+\alpha_{2}\right) a^{2}$ in Equations (3) and (4) can be ignored in the present data-fitting. That was exactly what we did; as a result, only three parameters, namely $m(\cong-2 / 3), \mu$, and $\alpha_{1}$, were left to be determined.

We can easily recast the data of McTigue and others in terms of the stretch ratio $\lambda(t) \equiv l(t) / L$; here $L$ is the original length of the specimen and $l(t)$ is its length at the instant $t$. By substituting $a=\dot{\lambda} / \lambda$ and ignoring the term $3\left(\alpha_{1}+\alpha_{2}\right) a^{2}$ in Equations (3) and (4), we obtain for models (I) and (II) the following second-order differential equations:

$$
\text { (I) } 3 \alpha_{1}\left(\ddot{\lambda} / \lambda-(\dot{\lambda} / \lambda)^{2}\right)+3 \mu\left(3(\dot{\lambda} / \lambda)^{2}\right)^{m / 2}(\dot{\lambda} / \lambda)-\sigma=0 \text {, }
$$

$$
\text { (II) } 3 \alpha_{1}\left(\ddot{\lambda} / \lambda-(\dot{\lambda} / \lambda)^{2}\right)+3 \mu \dot{\lambda} / \lambda-\left(3(\dot{\lambda} / \lambda)^{2}\right)^{-m / 2} \sigma=0 \text {. }
$$

The data-fitting problem for model (I) is as follows: for each test we are given a set of data points $\left(t_{\mathrm{i}}, \lambda_{\mathrm{i}}\right)$, where $t_{\mathrm{i}}=1,2, \ldots, 17 \mathrm{day}$, and $\lambda_{\mathrm{i}}$ is the measured value of $\lambda$ at $t_{\mathrm{i}}$. From the data we can determine the rate of stretching $\dot{\lambda}_{i}$ at $t_{1}$ by polynomial interpolation. For a given set of parameters $\left(m, \mu, \alpha_{1}\right)$, let $\lambda\left(t ; m, \mu, \alpha_{1}\right)$ be the solution to Equation (10) with initial conditions $\lambda(1 ; m, \mu$, $\left.\alpha_{1}\right)=\lambda_{1}$ and $\dot{\lambda}\left(1 ; m, \mu, \alpha_{1}\right)=\dot{\lambda}_{1}$. We want to determine values of the parameters, $m, \mu$, and $\alpha_{1}$ which minimize the function

$$
F\left(m, \mu, \alpha_{1}\right)=\sum_{1}\left(\lambda\left(t_{\mathrm{i}} ; m, \mu, \alpha_{1}\right)-\lambda_{\mathrm{i}}\right)^{2} .
$$

The data-fitting problem for model (II) is similar.

We solved the data-fitting problem for model (I) iteratively by using IMSL (International Mathematical and Statistical Library) sub-routine ZXSSQ (a finite-difference analogue of the Levenberg-Marquardt method). We arrived at a set of initial guesses for $\left(m, \mu, \alpha_{1}\right)$ by the following procedure: first we chose a value of $m$ close to $-2 / 3$. Using

*McTigue and others, who adopted the second-order fluid model, also encountered the problem of over-parameterization. We noticed that the estimated values of material parameters reported by McTigue and others for test 1 and test 4 as well as the reported means of the estimates for tests 2,3 , and 4 are inconsistent with their assumption that $\xi^{2}>0$ (see their equation (20) for the definition of $\xi^{2}$ ), which they use when they develop their theoretical solution equation (22)). There may be something wrong in their data-fitting. this value of $m$, we calculated the corresponding value of $\mu$ from Equation (8) and the measured value of $a_{\min }$. By polynomial interpolation of the given data, we estimated $\lambda$ and $\ddot{\lambda}$ at $t=2$. From these estimates, $\lambda_{2}$ and the first estimates of $m$ and $\mu$, we obtained a rough first estimate of $\alpha_{1}$ from Equation (10). To evaluate the function $F$ at each iteration, the corresponding values $\lambda\left(t_{\mathrm{j}} ; m, \mu, \alpha_{1}\right)$ are required. We obtain these values through numerical integration of Equation (10) by using the fifth-order Runge-Kutta-Nyström method (cf. Lambert, 1973, p. 122) Similarly, we solved the data-fitting problem for model (II). Complete details of the data-fitting with computer programs have been given by Sun (unpublished).

The problem of over-parameterization persists in the data-fitting even after the parameter $\alpha_{2}$ is dropped from the picture. For both models and for each test there are many combinations of $m(\cong-2 / 3), \mu$, and $\alpha_{1}$ which give essentially the same value to the sum of squared residuals $F$. For a given test and for a fixed value of $m$, however, the estimates of $\mu$ and $\alpha_{1}$ are sharp for both models. Moreover, when $m$ ranges over values close to $-2 / 3$, the scatter in the numerical estimates of $\mu$ and $\alpha_{1}$ is rather narrow. For instance, for test 1 , as $m$ ranges from -0.65 to -0.68 , we found that for model (I) $\mu$ ranges from $2.82 \times 10^{3}$ to $2.31 \times 10^{3} \mathrm{kPa} \mathrm{d}^{1+m}$, and $\alpha$ increases from $1.26 \times 10^{5}$ to $1.40 \times 10^{5} \mathrm{kPa} \mathrm{d}^{2}$; for model (II) $\mu$ decreases from $2.84 \times$ $10^{3}$ to $2.32 \times 10^{3} \mathrm{kPa} \mathrm{d}^{1+m}$, and $\alpha_{1}$ decreases from $2.75 \times$ $10^{3}$ to $2.03 \times 10^{3} \mathrm{kPa} \mathrm{d}^{2+m}$.

In this note we shall be content to obtain for models (I) and (II) an order-of-magnitude estimate of the normal stress effects discussed by McTigue and others. Moreover, we shall be interested only in situations where the normal stress effects have a sufficiently large magnitude to be practically significant. Since the data of McTigue and others all pertain to the same $\sigma$ and are not sufficient to give a sharp estimate of $m$, in what follows we shall take $m=-2 / 3$ (i.e. $n=3$ ) and estimate $\mu$ and $\alpha_{1}$ on that basis. While this specific choice of $m$ is somewhat arbitrary, it is our best choice and is certainly good enough for our present purpose; our conclusions below are insensitive to variations of $m$ near $-2 / 3$. For instance, as the reader can easily do the calculations himself, taking $m=-0.65$ or $m=-0.68$ and the corresponding values of $\mu$ and $\alpha_{1}$ (estimated from test 1) given above will not affect the qualitative conclusions to be drawn below where we take $m=-2 / 3$. Indeed, a sampling of other choices of $m$ in the range -0.65 to -0.71 all leads to the same qualitative conclusions; cf. Sun (unpublished).

The least-squares estimates of $\mu$ and $\alpha_{1}$ when $m$ is fixed at $-2 / 3$ are listed in Tables I and II. The scatter in the estimated values of the parameters is much narrower than that reported by McTigue and others. Under the estimated values of the material parameters, the fits are excellent for all four tests and for both models. The percentage errors in the fitted values of $\lambda$ as compared with the measured values are mostly under $\pm 0.02 \%$; the highest percentage errors are about $\pm 0.04 \%$.

Having obtained the value of $\mu$ for models (I) and (II), respectively, we can now look at estimates (6) and (7) in detail. Let us consider estimate (6) first. Within the range of octahedral shear stresses under which Glen's flow law is presumed to be valid (i.e. $0.1 \mathrm{MPa} \leqslant \tau \leqslant 1 \mathrm{MPa}$ ), the maximum value of $|a|_{\min }$ corresponds to $\tau=1 \mathrm{MPa}$. Substituting $\sigma=-3 / 2^{\frac{1}{2}} \mathrm{MPa}$ and $\mu=2.41 \times 10^{3} \mathrm{kPa} \mathrm{d}^{1 / 3}$, we calculate from Equation (8) that $|a|_{\min }=0.0758 \mathrm{~d}^{-1}$, for which the right-hand side of estimate $(6)$ is equal to $1.23 \times$ $10^{5} \mathrm{kPa} \mathrm{d}^{2}$. Since $\alpha_{1}$ is estimated to be equal to $1.61 \times 10^{5}$ $\mathrm{kPa} \mathrm{d}^{2}$, estimate (6) dictates that for model (I) $\left|\alpha_{1}+\alpha_{2}\right|$ should be at least an order of magnitude smaller than $\alpha_{1}$. Similarly, for model (II), corresponding to $\tau=1 \mathrm{MPa}$ and $\mu=2.43 \times 10^{3} \mathrm{kPa} \mathrm{d}^{1 / 3}$, the right-hand side of estimate (7) is equal to $3.29 \times 10^{4} \mathrm{kPa} \mathrm{d}^{4 / 3}$. Thence, we infer that for model (II) $\left|\alpha_{1}+\alpha_{2}\right|$ should be at most of the same order of magnitude as $\alpha_{1}$.

\section{NORMAL STRESS DIFFERENCES IN SHEARING FLOW}

Both models (I) and (II) can exhibit non-zero and unequal normal stress differences in shearing flow (cf. 
Schowalter (1978, p. 71) for the definition of normal stress differences). For models (I) and (II), respectively, the normal stress differences are given by

$$
\begin{gathered}
\text { (I) } N_{1}=-2 \alpha_{1} \mathrm{k}^{2}, \quad N_{2}=\left(2 \alpha_{1}+\alpha_{2}\right) \mathrm{k}^{2}, \\
\text { (II) } N_{1}=-2 \alpha_{1} \mathrm{k}^{2+m}, \quad N_{2}=\left(2 \alpha_{1}+\alpha_{2}\right) \mathrm{k}^{2+m} ;
\end{gathered}
$$

here $k$ is the shear rate. The formulae for model (I) will be identical to those for the second-order fluid (McTigue and others, 1985) if we replace $\alpha_{1}$ and $\alpha_{2}$ in Equation (13) by the parameters $\mu_{2}$ and $\mu_{3}$ of McTigue and others. It is thus possible to compare directly the magnitudes of $N_{1}$ and $N_{2}$ according to our model (I) with those reported by McTigue and others for the second-order fluid model

For model (I), by using the mean value of $\alpha_{1}$ in Table $I$ and by changing units, we obtain

$$
N_{1}=-2.40 \times 10^{18} \mathrm{\kappa}^{2} \mathrm{~Pa}
$$

where the shear rate $k$ is in units of $s^{-1}$. Since all that we know about the parameter $\alpha_{2}$ is the estimate (6), we can at best give an order-of-magnitude estimate of $N_{2}$. Since $\left|\alpha_{1}+\alpha_{2}\right|$ is estimated to be at least an order of magnitude smaller than $\alpha_{1},\left|N_{2}\right|$ will have the same order of magnitude as $\left|N_{1}\right|$.

For the second-order fluid model, McTigue and others found that $N_{1}=2.1 \times 10^{19} \mathrm{k}^{2} \mathrm{~Pa}$ and $N_{2}=3.4 \times 10^{21} \mathrm{\kappa}^{2} \mathrm{~Pa}$ (cf. their equations (23a) and (23b)). Their value of $N_{1}$ is one order of magnitude bigger than the first normal stress difference in our model (I); moreover, it has a different sign. Their value of $\mathrm{N}_{2}$ is three orders of magnitude bigger than that in our model (I).

The tremendous discrepancy in the estimates of normal stress differences, we believe, is due in part to the problem of over-parameterization in the data-fitting. As noted by McTigue and others, "good fits ... to the creep data can be found for broad ranges of [the] parameters $\left[\mu_{2}\right.$ and $\left.\mu_{3}\right]$ ". We overcame the problem of over-parameterization by fixing the value of $m$ at $-2 / 3$ (i.e. $n=3$ ) and by ignoring in Equations (3) and (4) the term $3\left(\alpha_{1}+\alpha_{2}\right) a^{2}$, which should be negligible for consistency with Glen's flow law. Working with the second-order fluid model is like setting $m=0$ in our models at the outset, but there is no longer Glen's flow law to lean on, for the second-order fluid model is inconsistent with Glen's flow law. There does not seem to be any convincing way to evade the problem of over-parameterization when one attempts to fit the data of McTigue and others to the second-order fluid model.

Over-parameterization was not a problem in the pressuremeter study of Man and the Manitoba team on polycrystalline ice at $-2^{\circ} \mathrm{C}$ (Man and others, 1985; K jartanson, unpublished; Sun, unpublished). For both models (I) and (II), the parameter $\alpha_{2}$ simply does not appear in the respective differential equation that governs pressuremeter creep tests. With data of single-stage and multi-stage creep tests in which the cavity pressure ranged from 1.0 to 2.5 $\mathrm{MPa}$ and the early time responses were recorded in detail, the parameters $m, \mu$, and $\alpha_{1}$ could be determined unambiguously for both models. For the specimens of ice they studied, they found that $m$ was indeed close to $-2 / 3$ and $\alpha_{1}$ was positive for both models. For model (I), the value of $\alpha_{1}$ was estimated to be $9.17 \times 10^{14} \mathrm{~Pa} \mathrm{~s}{ }^{2}$, for which $N_{1}=-1.83 \times 10^{15} \mathrm{k}^{2} \mathrm{~Pa}$. The discrepancy between Equation (15) and this estimate of $N_{1}$ results from the corresponding difference between the value of $\alpha_{1}$ given earlier in Table I and the value estimated from the pressuremeter tests. For a discussion on the possible implications of the latter difference, of. the remark at the end of the next section.

FREE-SURFACE DEPRESSION (OR HEAVE) IN OPENCHANNEL FLOW

McTigue and others made use of an approximation scheme (due to Wineman and Pipkin (1966), and Tanner (1970); cf. also Schowalter (1978, p. 255-57)) to calculate the free-surface depression or heave of a second-order fluid when it flows steadily down an inclined open semi-circular channel. Using the values of material parameters which they estimated from their creep tests, they applied their surface-depression formula to the flow of glaciers.

The free-surface depression or heave studied by McTigue and others is due to a non-vanishing second normal-stress difference $N_{2}$. Both models (I) and (II) can also exhibit the same phenomenon. Indeed, by following the same approximation procedure, it is straightforward to work out the corresponding formulae for these two models. In our derivation, the usual power-law fluid assumes a role parallel to that of the Newtonian fluid in the derivation of McTigue and others.

Let $\rho$ be the density of ice, $g$ be the acceleration due to gravity, $h$ be the central (maximum) rise or fall, $\beta$ be the channel slope, and $R$ be the channel radius (see figure 3 of McTigue and others). The formulae in question are found for models (I) and (II), respectively, to be as follows:

$$
\text { (I) } h=-\left(2 \alpha_{1}+\alpha_{2}\right) \frac{(3+m)}{2 \rho g \cos \beta}\left[\frac{R \rho g \sin \beta}{2 \mu}\right)^{2 /(1+m)} \text {; }
$$

(II) $h=-4\left(2 \alpha_{1}+\alpha_{2}\right) \frac{(3+2 m)}{(2+m) \rho g \cos \beta}\left[\frac{R \rho g \sin \beta}{4 \mu}\right]^{(2+m) /(1+m)}$

When $m=0$, both Equations (16) and (17) reduce to the formula for the second-order fluid, i.e. equation (26) of McTigue and others. For the usual power-law fluid, which is the special instance of our models with $\alpha_{1}=\alpha_{2}=0$, both Equations (16) and (17) give $h=0$.

Earlier we have argued that for model (I) $\left|\alpha_{1}+\alpha_{2}\right|$ should be at least an order of magnitude smaller than $\alpha_{1}$; for model (II) $\left|\alpha_{1}+\alpha_{2}\right|$ should at most have the same order of magnitude as $\alpha_{1}$. For a given $R$ and $\beta$, we can easily obtain an order-of-magnitude estimate of $|h|$ for models (I) and (II). Let us consider model (I). First, we set $\alpha_{1}+\alpha_{2}=0$. The right-hand side of Equation (16) can then be evaluated. Let us denote the resulting value by $h_{0}$. Since $\left|\alpha_{1}+\alpha_{2}\right|$ is at least an order of magnitude smaller than $\alpha_{1},\left|h_{0}\right|^{2}$ and $|h|$ should have the same order of magnitude. Similarly, for a given $R$ and $\beta$, we infer that for model (II)

$|h|$ can at most have the same order of magnitude as $\left|h_{0}\right|$. Let us evaluate $\left|h_{0}\right|$ for some sample channel slopes and channel radii. We take $\rho=9 \times 10^{2} \mathrm{~kg} / \mathrm{m}^{3}$, $g=9.8 \mathrm{~m} / \mathrm{s}^{2}, \quad m=-2 / 3$, and give $\mu$ and $\alpha_{1}$ the mean values given in Tables I and II. For $\beta=10^{\circ}$ and $R=500 \mathrm{~m}$, we obtain the following values for models (I)

TABLE I. LEAST-SQUARES ESTIMATES OF $\mu$ AND $\alpha_{1}$ FOR MODEL (I) WHEN $m$ IS FIXED AT $-2 / 3$

$\begin{array}{ccc}\text { Test } & \mu & \alpha_{1} \\ & 10^{3} \mathrm{kPa} \mathrm{d}^{1 / 3} & 10^{5} \mathrm{kPa} \mathrm{d}^{2} \\ 1 & 2.52 & 1.33 \\ 2 & 2.54 & 2.01 \\ 3 & 2.33 & 1.13 \\ 4 & 2.26 & 1.98 \\ & & \\ \text { Mean } & 2.41 & 1.61\end{array}$

TABLE II. LEAST-SQUARES ESTIMATES OF $\mu$ AND $\alpha_{1}$ FOR MODEL (II) WHEN $m$ IS FIXED AT $-2 / 3$
Test

2

3

Mean $\mu$

$10^{3} \mathrm{kPa} \mathrm{d}^{1 / 3}$

2.53

2.58

2.34

2.29

2.43 $\alpha_{1}$ $10^{3} \mathrm{kPa} \mathrm{d}^{4 / 3}$
4.16 
and (II), respectively: (I) $\left|h_{0}\right|=0.35 \mathrm{~m}$; (II) $\left|h_{0}\right|=0.07 \mathrm{~m}$. For the same $\beta$ and $R$, McTigue and others estimated $h=$ $-42 \mathrm{~m}$. For $B=5^{\circ}$ and $R=250 \mathrm{~m}$, McTigue and others obtained $h=-2.6 \mathrm{~m}$; for models (I) and (II) we get (I) $\left|h_{0}\right|=0.08 \mathrm{~mm}$, and (II) $\left|h_{0}\right|=0.26 \mathrm{~mm}$.

For a given $R$ and $\beta$, let $h^{*}$ be the value of $h$ estimated by McTigue and others. In general $\left|h^{*}\right|$ is related to $\left|h_{0}\right|$ of models (I) and (II) by what follows:

$$
\begin{aligned}
& \text { (I) }\left|h_{0}\right| /\left|h^{*}\right|=1.4 \times 10^{-10}(R \sin B)^{4} \text {; } \\
& \text { (II) }\left|h_{0}\right| /\left|h^{*}\right|=2.1 \times 10^{-7}(R \sin B)^{2} ;
\end{aligned}
$$

here $R$ is in units of meters. For the usual ranges of $R$ and $B$ of glaciers on Earth, the free-surface depression or heave according to both of our models is much smaller in magnitude than the prediction of McTigue and others. Indeed, according to either model (I) or (II) (with vaiues of material parameters given in Tables I and II), the freesurface depression or heave induced by the second normalstress difference will usually be negligible for glaciers.

For both models (I) and (II), the velocity field in the flow under consideration is, to the order of approximation adopted in the derivation of Equations (16) and (17), identical to that which pertains to Glen's flow law. For both models, the stress distribution is different from that of the usual power-law fluid. With the values of material parameters adopted above, however, the difference in stress distribution is found to be negligible for the usual ranges of $R$ and $B$ for glaciers. Thus, we cannot accept the argument of McTigue and others as regards the possible significance of normal stress effects in the formation of crevasses.

Remark. The values of $\left|h_{0}\right|$ are even smaller if we use values of $m, \mu$, and $\alpha_{1}$ estimated by Man and the Manitoba team from pressuremeter creep tests on polycrystalline ice at $-2^{\circ} \mathrm{C}$. The estimates were as follows (cf. Sun, unpublished): $\quad m=-0.711, \quad \mu=2.97 \times 10^{7} \mathrm{~Pa} \mathrm{~s}^{1+m} ; \quad$ for model (I), $\alpha_{1}=9.17 \times 10^{14} \mathrm{~Pa} \mathrm{~s}^{2}$; for model (II), $\alpha_{1}=2.46$ $\times 10^{10} \mathrm{~Pa} \mathrm{~s}^{2+m}$. For $\beta=10^{\circ}$ and $R=500 \mathrm{~m}$, it follows that for model (I) $\left|h_{0}\right|=9.8 \mathrm{~mm}$ and for model (II) $\left|h_{\rho}\right|=$ $2.3 \mathrm{~mm}$. For $\beta=5^{\circ}$ and $R=250 \mathrm{~m},\left|h_{0}\right|=0.7 \times 10^{-3} \mathrm{~mm}$ for model (I), and $\left|h_{0}\right|=4.8 \times 10^{-3} \mathrm{~mm}$ for model (II). These values of $\left|h_{0}\right|$ are about 30 to 100 times smaller than those calculated above. Do these discrepancies in prediction indicate a basic flaw in the modeling, namely, that different tests would deliver different values of the coefficients $m, \mu$, and $\alpha_{1}$ ? Probably not. In fact, there are encouraging signs. Recall that for both models (I) and (II) $m$ and $\mu$ should be related to the coefficients $n$ and $B$ in Glen's law through Equation (9). Many researchers have performed uniaxial compression tests on ice specimens at various temperatures. A range of values of $n$ and $B$ (and thus also of $m$ and $\mu$ ) has been reported in the literature. The values of $m$ and $\mu$ estimated from the pressuremeter tests are in good agreement with what other researchers have found from uniaxial compression tests (cf. K jartanson and others, in press). The values of $\mu$ given in Tables I and II also fall in the range of values reported in the literature (cf. Hooke, 1981, fig. 2). Of course, since $B$ and thence also $\boldsymbol{\mu}$ vary appreciably with temperature, when we compare the values of $\mu$, comparison is made only among those values that pertain to the same temperature. A problem remains with the coefficient $\alpha_{1}$. The discrepancy is enormous between the values of $\alpha_{1}$ reported earlier in this paragraph and those given in Tables I and II (i.e. $\alpha_{1}=$ $1.20 \times 10^{18} \mathrm{~Pa} \mathrm{~s}^{2}$ for model (I); $\alpha_{1}=1.15 \times 10^{13} \mathrm{~Pa} \mathrm{~s}^{4 / 3}$ for model (II)). To clarify this point, more tests are necessary. Before results of new tests are available, allow us to do some speculation. Of all factors that could possibly account for this discrepancy (for instance, the specimens of the pressuremeter tests would have structural features different from those of the triaxial tests), temperature would be the most interesting. If temperature should be the main source of the discrepancy, then the significant difference in the estimated values of $\left|h_{0}\right|$ could be attributed to the difference in temperature between the pressuremeter tests $\left(-2^{\circ} \mathrm{C}\right)$ and the triaxial tests $\left(-9.5\right.$ to $\left.-9.8^{\circ} \mathrm{C}\right)$. In other words, lowering of temperature might greatly enhance normal stress effects in the creep of ice.

\section{CONCLUSION AND DISCUSSION}

McTigue and others (1985) identified a possible problem in the type of constitutive equation usually used for modeling the creep behavior of polycrystalline ice. They pointed out that Glen's flow law necessarily excludes the consideration of normal stress effects, which are of great significance in other disciplines that consider non-Newtonian fluids. Using the second-order fluid (with material parameters evaluated from triaxial creep tests) as a tentative model for ice, they reached the conclusion that normal stress effects may be discernible in natural glacier flow. The argument which led them to the preceding conclusion, however, can be challenged on the following grounds: (i) As noted by McTigue and others themselves, the second-order fluid "fails to represent the non-linear rate dependence of ice in shear"; therefore, it is in fact not a suitable constitutive model for glacier ice in shearing flow. The creep tests with which they quantified the material parameters in the second- order fluid were performed at the same deviatoric stress. Had they used data of creep tests which pertained to a range of deviatoric stresses, they would have had a hard time in finding a single set of parameters that could fit the data. (ii) The conclusion of McTigue and others naturally depends very much on the numerical values of material parameters which they put in the second-order fluid model. They obtained those numerical values by fitting data of their creep tests. Their data analysis, however, seemingly had inconsistencies (cf. footnote above). Moreover, they did not indicate how they handled the problem of overparameterization in their data-fitting.

The preceding criticism is not meant to play down the contribution of McTigue and others. They have identified and asked an important question, which, as they fully realize, has no simple immediate answer. They concluded their paper by envisaging that their results should "serve as a point of departure for further investigation", to which our present note bears witness.

In this note we have re-examined the issues discussed by McTigue and others in the light of two new tentative models, namely (I) the modified second-order fluid and (II) the power-law fluid of grade 2, both of which can be taken as simple modifications of Glen's flow law. For ease of comparison with the work of McTigue and others, we have used the same creep data in quantifying the material parameters in models (I) and (II). As indicated above, there is a problem of over-parameterization in the data-fitting, and we have shown how we can circumvent that problem to obtain order-of-magnitude estimates for the normal stress effects discussed by McTigue and others. For both models (I) and (II), the normal stress effects are found to be far less pronounced than the estimates of McTigue and others. Indeed, should glacier ice have creep properties similar to the specimens of McTigue and others, models (I) and (II) indicate that, except for extremely thick ice and steep slopes, normal stress effects of the type discussed by McTigue and others would not play a significant role in glacier flow.

The preceding conclusion remains valid, should we use values of material parameters estimated by Man and the Manitoba team from data of pressuremeter creep tests on polycrystalline ice at $-2{ }^{\circ} \mathrm{C}$ (Man and others, 1985; $\mathrm{Kjartanson}$, unpublished; Sun, unpublished); cf. the remark at the end of the preceding section. The problem of overparameterization did not appear in their pressuremeter work. Pressuremeter creep tests, however, can only deliver estimates for the parameters $m, \mu$, and $\alpha_{1}$. As indicated above, by appealing to Glen's law, we do not need to know the value of $\alpha_{2}$ to obtain an order-of-magnitude estimate of the normal stress effects discussed in this note. On the other hand, procurement of experimental data that suffice for the estimation of all the material parameters will not only tighten our argument; it will also provide a more stringent test for the models. For a given ice specimen, it should be possible to obtain the values of $m, \mu, \alpha_{1}$, and $\alpha_{2}$ for models (I) and (II) by performing both pressuremeter and triaxial creep tests which record early time response in detail.

A correct assessment of the significance of normal 
stress effects in glacier flow can be made only after a sufficiently accurate flow law is established and values of material parameters in the flow law are ascertained for various ice forms and temperatures. At present it is premature on both counts to give a sweeping conclusion. Models (I) and (II) are still tentative. A lot more theoretical and experimental work remains to be done before we can clarify their status. Even if one of them turns out to be a good model for ice in creep, our analysis above still does not necessarily imply that normal stress effects can be ignored when we consider the creep behavior of polycrystalline ice. For instance, as pointed out in the preceding section, lowering of temperature might greatly enhance normal stress effects in the creep of ice.

\section{ACKNOWLEDGEMENTS}

We are indebted to Dr S.J. Jones, National Research Council of Canada, who kindly sent us and granted us permission to use his data of triaxial creep tests, without which this note could not have been written. We are grateful to Dr S.L. Passman for sending us a preprint of his joint work with McTigue and Jones. We thank Dr D.F. McTigue, whose comments on an earlier draught of this note have led to improvement of our presentation in several places. The present work was initiated when C.-S. Man was Assistant Professor of Civil Engineering at the University of Manitoba. There, his researches were supported by grants from the Natural Sciences and Engineering Research Council of Canada. C.-S. Man did part of the work reported here when he was a long-term visitor at the Institute for Mathematics and its Applications, University of Minnesota. There, related to data-fitting in the present work, he had a helpful conversation with Professor G.R. Sell. The final revision of the manuscript was supported in part with funds from the U.S. National Science Foundation (grant No. RII8610671 ) and the Commonwealth of Kentucky through the Kentucky EPSCoR Program. The publication of this note was supported by the U.S. National Science Foundation under grant DMS-8703731.

\section{REFERENCES}

Bird, R.B., and others. 1977. Dynamics of polymeric liquids. Vol. 1. By R.B. Bird, R.C. Armstrong, and O. Hassager. New York, etc., Wiley.

Coleman, B.D., and Noll, W. 1960. An approximation theorem for functionals, with applications in continuum mechanics. Archive for Rational Mechanics and Analysis, Vol. 6, No. 5, p. 355-70.

Dunn, J.E. 1982. On the free energy and stability of nonlinear fluids. Journal of Rheology, Vol. 26, No. 1, p. 43-68.

Glen, J.W. 1952. Experiments on the deformation of ice. Journal of Glaciology, Vol. 2, No. 12, p. 111-14.
Glen, J.W. 1955. The creep of polycrystalline ice. Proceedings of the Royal Society of London, Ser. A, Vol. 228, No. 1175, p. 519-38.

Hooke, R.L. 1981. Flow law for polycrystalline ice in glaciers: comparison of theoretical predictions, laboratory data, and field measurements. Reviews of Geophysics and Space Physics, Vol. 19, No. 4, p. 664-72.

Kjartanson, B.H. Unpublished. Pressuremeter creep testing in laboratory ice. [Ph.D. thesis, University of Manitoba, 1986.]

Kjartanson, B.H., and others. In press. The creep of ice measured with the pressuremeter, by B.H. K jartanson, D.H. Shields, L. Domaschuk, and C.-S. Man. Canadian Geotechnical Journal.

Lambert, J.D. 1973. Computational methods in ordinary differential equations. London, etc., Wiley.

McTigue, D.F., and others. 1985. Normal stress effects in the creep of ice, by D.F. McTigue, S.L. Passman, and S.J. Jones. Journal of Glaciology, Vol. 31, No, 108, p. 120-26.

Man, C.-S., and others. 1985. Creep of ice as a fluid of complexity. 2: the pressuremeter problem, by C.-S Man, D.H. Shields, B. K jartanson, and Q.-X. Sun. (In Rasmussen, H., ed. Proceedings of the Tenth Canadian Congress of Applied Mechanics, London, Ontario, June 2-7. 1985. Vol. 1, p. A347-48.)

Morland, L.W., and Spring, U. 1981. Viscoelastic fluid relation for the deformation of ice. Cold Regions Science and Technology, Vol. 4, No. 3, p. 255-68.

Nye, J.F. 1952. The mechanics of glacier flow. Journal of Glaciology, Vol. 2, No. 12, p. 82-93.

Nye, J.F. 1953. The flow law of ice from measurements in glacier tunnels, laboratory experiments and the Jungfraufirn borehole experiment. Proceedings of the Royal Society of London, Ser. A, Vol. 219, No. 1139, p. 477-89.

Nye, J.F. 1957. The distribution of stress and velocity in glaciers and ice-sheets. Proceedings of the Royal Society of London, Ser. A, Vol. 239, No. 1216, p. 113-33.

Paterson, W.S.B. 1981. The physics of glaciers. Second edition. Oxford, etc., Pergamon Press. (Pergamon International Library.)

Rivlin, R.S., and Ericksen, J.L. 1955. Stress-deformation relations for isotropic materials. Journal of Rational Mechanics and Analysis, Vol. 4, p. 323-425.

Schowalter, W.R. 1978. Mechanics of non-Newtonian fluids. Oxford, etc., Pergamon Press.

Sun, Q.-X. Unpublished. On two special Rivlin-Ericksen fluid models generalizing Glen's flow law for polycrystalline ice. [Ph.D. thesis, University of Manitoba, 1987.]

Tanner, R.I. 1970. Some methods for estimating the normal stress functions in viscometric flows. Transactions of the Society of Rheology, Vol. 14, No. 4, p. 483-507.

Truesdell, C., and Noll, W. 1965. The non-linear field theories of mechanics. (In Flügge, S., ed. Encyclopedia of physics. Vol. III, Pt. 3. Berlin, etc., Springer-Verlag.)

Wineman, A.S., and Pipkin, A.C. 1966. Slow viscoelastic flow in tilted troughs. Acta Mechanica, Vol. 2, p. 104-15. 